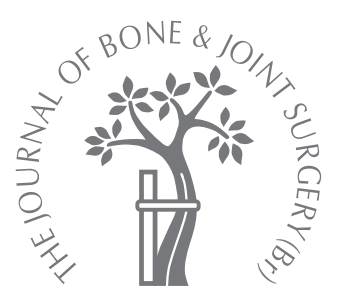

K. K. Park,

C. B. Chang,

Y. G. Kang,

S. C. Seong,

T. K. Kim

From Joint

Reconstruction

Center, Seoul

National University

Bundang Hospital,

Seoul, Korea

\title{
Correlation of maximum flexion with clinical outcome after total knee replacement in Asian patients
}

\footnotetext{
K. K. Park, MD, Clinical Fellow

I C. B. Chang, MD, PhD, Assistant Professor

= Y. G. Kang, BS, Clinical Investigator

" T. K. Kim, MD, PhD, Assistant Professor

Joint Reconstruction Center

Seoul National University

Bundang Hospital, 300 Gumi-

dong, Bundang-gu, Seongnam-

si, Gyeonggi-do (463-707),

Seoul, Korea.

= S. C. Seong, MD, PhD,

Professor

Seoul National University

Hospital, 28 Yeongon-dong,

Chongno-gu, Seoul, Korea.

Correspondence should be sent to Dr T. K. Kim; e-mail:

osktk@snubh.org
}

(C)2007 British Editorial Society of Bone and Joint Surgery doi:10.1302/0301-620X.89B5. $18117 \$ 2.00$

$J$ Bone Joint Surg [Br] 2007;89-B:604-8.

Received 10 May 2006;

Accepted after revision

19 January 2007

This study aimed to determine the correlation between the amount of maximum flexion and the clinical outcome in 207 Koreans (333 knees) undergoing total knee replacement. The association of maximum flexion with clinical outcome was evaluated one year postoperatively using three scoring systems; the American Knee Society score, Western Ontario McMaster Universities Osteoarthritis index and the Short Form-36. The mean maximum flexion decreased post-operatively at 12 months from $140.1^{\circ}\left(60^{\circ}\right.$ to $\left.160^{\circ}\right)$ to $133.0^{\circ}\left(105^{\circ}\right.$ to $150^{\circ}$ ). Only the social function score of the Short Form-36 correlated significantly with maximum flexion (correlation coefficient $=0.180, p=0.039$ ). In comparative analyses of subgroups divided by a maximum flexion of $120^{\circ}$, we found no significant differences in any parameters except the social function score of the Short Form-36 (41.9 vs 47.3, $p=0.031$ ). Knees with a maximum flexion of more than $135^{\circ}$ had a better functional Western Ontario McMasters Universities Osteoarthritis index score than knees with maximum flexion of $135^{\circ}$ or less (17.5 vs $14.3, p=0.031$ ). We found only weak correlation between the postoperative maximum flexion and the clinical parameters for pain relief, function and quality of life, even in Korean patients. Efforts to increase post-operative maximum flexion should be exercised with caution until concerns relating to high-flexion activities are sufficiently resolved.

Maximum flexion is widely used as an outcome measure after total knee replacement (TKR), and is a major component of many scoring systems. ${ }^{1,2}$ Values around $110^{\circ}$ are sufficient to perform most daily activities, including walking, climbing stairs and rising from a chair. $^{3-5}$ In most current reports, maximum flexion is greater than in earlier series. ${ }^{6-14}$ Despite this improvement, which typically ranges between $100^{\circ}$ and $125^{\circ}$, activities requiring flexion beyond $120^{\circ}$ are often impossible after TKR. ${ }^{4,5,7,15-17}$

With the success of current implants, the expectations of patients and surgeons for improved function after TKR have increased dramatically. ${ }^{4,5,9,14,18-20}$ Full flexion is often necessary in Asian culture to perform key routine activities such as kneeling, squatting and sitting with both legs crossed, in religious groups and in Western leisure activities such as gardening and bathing. ${ }^{5,18,21}$ In response to such demand, efforts have been made to achieve greater flexion after TKR. ${ }^{9,13,14,18,20,22}$

Whether this brings real benefit, however, is a cause for debate. ${ }^{13,14}$ Some reports of current TKR systems which provide greater flexion and better kinematic patterns suggest that activities requiring full flexion would be possible without threatening survival of the implant. ${ }^{9,20,23,24}$ Conversely, others have found no significant clinical advantages with highflexion designs. ${ }^{13,14}$

It is reasonable to believe that greater flexion after TKR brings a better clinical outcome. However, there are few studies which investigate the correlation between maximum flexion and clinical outcome, ${ }^{12,25}$ and, particularly, none in Asians. ${ }^{21}$ We therefore undertook such a study with the hypothesis that the post-operative maximum flexion would correlate with clinical outcome in Korean patients, who frequently perform full-flexion activities.

\section{Patients and Methods}

We evaluated 350 TKRs performed in 220 consecutive osteoarthritic patients between November 2003 and February 2005. Six patients (seven knees) were excluded: three patients (three knees) had peri-prosthetic infection, one patient (two knees) died in circumstances unrelated to surgery, one patient (one knee) had a cerebrovascular accident and one patient (one knee) had severe spinal problems. A further seven patients (ten knees) were 
excluded as they did not attend the clinic. Consequently, 333 knees (207 patients) which were followed up beyond 12 months were included. A total of 81 TKRs were unilateral and 126 were bilateral, with an interval of between one and three weeks (typically two) between operations. There were 196 women and 11 men with a mean age of 68.7 years (54 to 87). The mean height and weight were $151.6 \mathrm{~cm}$ (138 to 174 ) and $60.4 \mathrm{~kg}$ (36 to 83 ). The mean body mass index (BMI) was $26.2 \mathrm{~kg} / \mathrm{m}^{2}$ (18.0 to 37.0 ). All patients gave informed consent to participate in the study.

Pre-operative information was gathered three days before surgery by an independent investigator (YGK), who recorded the range of movement (ROM), flexion contracture and the maximum flexion, and collected data from questionnaires. The pre-operative evaluation included the demography, diagnosis, medical history and physical examination, including the ROM, and collateral and anteroposterior stress tests. The passive, non-weight-bearing ROM was measured to the nearest $5^{\circ}$ by a goniometer, with the patient supine. Patients were also evaluated by the knee and function scores of the American Knee Society (AKS), ${ }_{1}^{1}$ Western Ontario McMaster Universities Osteoarthritis Index (WOMAC) ${ }^{26}$ and the Short Form-36 (SF-36). ${ }^{27}$

All operations were performed by a single surgeon (TKK). A total of 172 knees were implanted with E-motion (FP type, Aesculap, Tuttlingen, Germany) and 161 with Genesis II (CR type in 112 knees and PS type in 49; Smith $\&$ Nephew, Memphis, Tennessee) arthroplasties. All operations used a medial parapatellar arthrotomy, and for 101 TKRs with the E-motion, computer-assisted navigation was used (Orthopilot 4.0, Aescalup, Germany). The other 71 TKRs with the E-motion and 161 with Genesis II, used conventional intramedullary guides for the femur and extramedullary guides for the tibia. Patellar resurfacing was routine and all implants were fixed with cement.

The patients remained in hospital for two weeks, undergoing a standard rehabilitation programme, commencing on the first post-operative day with continuous passive movement. On the third day, they attended the rehabilitation department for training with crutches or walking aids. Between the fourth and the 14th days they had daily physiotherapy. Progression to maximum flexion was recorded with the goal of achieving $110^{\circ}$ of flexion without a flexion contracture by discharge.

Post-operative assessment was carried out by the same investigator (YGK) at three, six and 12 months, and yearly thereafter. Evaluations included ROM, AKS, WOMAC and SF-36 scores. In bilateral cases, AKS and WOMAC scores were measured separately for each knee.

Statistical analysis was performed using SPSS for Windows (version 12.0, SPSS Inc., Chicago, Illinois) with a p-value $<0.05$ considered significant. The relationship between maximum flexion and the clinical scores was investigated by correlation analysis. In bilateral cases, the lesser maximum flexion was used in the analyses for SF-36. In order to adjust the effects of confounding factors, maximum flexion was analysed for its association with age, gender, weight, height, BMI, category of AKS (A, unilateral or bilateral with opposite knee successfully replaced; B, unilateral with other knee symptomatic; C, multiple arthritis or medical infirmity) and type of implant. The final analyses were performed with the partial correlation test as the possible confounders were controlled. This was on the basis of the correlation coefficient (CC), being almost perfect $(0.81$ to 1.00$)$, excellent ( 0.61 to 0.80$)$, good or moderate $(0.41$ to 0.60$)$, fair $(0.21$ to 0.40$)$ or slight $(0.00$ to 0.20$) .^{28}$

In order to determine whether knees with maximum flexion allowing full-flexion activities had a better clinical outcome, comparative analyses between two pairs of the groups divided by the maximum flexion of $120^{\circ}$ and $135^{\circ}$ were carried out using Student's $t$-test. Possible confounding factors (gender, age, height, weight, BMI, type of implant, pre-operative scores of AKS, WOMAC, and SF36, unilateral or bilateral TKR, and patient category by AKS) were compared using the chi-squared test or Student's $t$-test. For those factors showing significant differences or statistical tendency $(\mathrm{p}<0.1)$, we carried out adjustment by the analysis of covariance (ANCOVA) for the numerical variables, and subgroup analyses for the categorical variables.

\section{Results}

The mean ROM (flexion contracture to maximum flexion) was $128.1^{\circ}\left(45^{\circ}\right.$ to $\left.160^{\circ}\right)$ pre-operatively and improved to $130.6^{\circ}\left(100^{\circ}\right.$ to $\left.150^{\circ}\right)$ at six months and $132.9^{\circ}\left(105^{\circ}\right.$ to $150^{\circ}$ ) at 12 months post-operatively. In contrast, the mean maximum flexion decreased from $140.1^{\circ}\left(60^{\circ}\right.$ to $\left.160^{\circ}\right)$ preoperatively to $133.0^{\circ}\left(105^{\circ}\right.$ to $\left.150^{\circ}\right)$ at 12 months postoperatively. Most of the clinical parameters improved significantly with time (Table I).

In the preliminary search for confounding factors for correlation analyses, the weight, BMI and type of implant (mean maximum flexion: Genesis II CR < E-motion < Genesis II PS) were associated with the maximum flexion. The subsequent correlation analyses were performed with these variables adjusted. Correlation analyses of the maximum flexion and pre-operative and clinical parameters revealed that only AKS had significant correlation (CC $=0.181$, $p=0.006)$. In the analyses of the parameters 12 months post-operatively, only the social functioning score of SF-36 had significant correlation $(\mathrm{p}=0.039)$, but the strength of that correlation was slight $(C C=0.180)$. The knee score of AKS and the function score of WOMAC showed a slight correlation with statistical tendency $(\mathrm{CC}=0.121, \mathrm{p}=0.077$ and $\mathrm{CC}=-0.129, \mathrm{p}=0.059$, respectively). No other parameters correlated significantly with the post-operative maximum flexion.

To determine whether knees with maximum flexion allowing high-flexion activities had a better clinical outcome, comparisons were made between two pairs of the groups divided by the maximum flexion of $120^{\circ}$ and $135^{\circ}$. The proportions of the knees with maximum flexion $>120^{\circ}$ 
Table I. Longitudinal data of the clinical outcomes using American Knee Society (AKS), Western Ontario and McMaster Osteoarthritis index (WOMAC), and Short Form (SF-36)

\begin{tabular}{|c|c|c|c|c|}
\hline \multirow[b]{2}{*}{ Parameter (maximum score) } & \multicolumn{4}{|c|}{ Mean score (SD) ${ }^{*}$} \\
\hline & Pre-operative & $\begin{array}{l}\text { Post-operative } \\
3 \text { months }\end{array}$ & $\begin{array}{l}\text { Post-operative } \\
6 \text { months }\end{array}$ & $\begin{array}{l}\text { Post-operative } \\
12 \text { months }\end{array}$ \\
\hline \multicolumn{5}{|l|}{ AKS } \\
\hline Pain $(50)$ & $22.7(6.1)$ & $46.1(3.9)$ & $46.7(4.7)$ & $47.9(5.2)$ \\
\hline Knee score (100) & $46.6(9.8)$ & $93.3(9.2)$ & $94.0(6.6)$ & $95.2(7.0)$ \\
\hline Function score (100) & $53.4(10.8)$ & $78.0(15.9)$ & $86.9(14.0)$ & $94.4(9.7)$ \\
\hline \multicolumn{5}{|l|}{ WOMAC $^{\dagger}$} \\
\hline Pain (20) & $11.5(4.3)$ & $5.4(4.1)$ & $3.5(3.6)$ & $2.2(2.8)$ \\
\hline Stiffness (8) & $4.7(2.1)$ & $3.0(1.7)$ & $2.4(1.7)$ & $1.5(1.5)$ \\
\hline Function (68) & $40.8(12.4)$ & $26.9(12.7)$ & $20.2(12.7)$ & $16.2(10.6)$ \\
\hline \multicolumn{5}{|l|}{ SF-36 } \\
\hline Physical functioning & $24.0(7.0)$ & $33.3(9.5)$ & $36.4(8.9)$ & $40.4(9.6)$ \\
\hline Role physical & $29.0(8.1)$ & $35.3(8.7)$ & $38.2(11.5)$ & $43.0(11.2)$ \\
\hline Bodily pain & $29.2(7.4)$ & $39.4(8.7)$ & $45.6(10.0)$ & $48.1(11.7)$ \\
\hline General health & $40.8(9.0)$ & $43.0(8.1)$ & $42.9(8.0)$ & $44.4(9.6)$ \\
\hline Vitality & $39.2(8.6)$ & $42.7(9.1)$ & $44.7(9.8)$ & $46.2(9.6)$ \\
\hline Social functioning & $33.9(11.2)$ & 37.9 (11.3) & $43.7(11.7)$ & $46.4(11.1)$ \\
\hline Role emotion & $30.8(12.7)$ & $33.8(13.5)$ & $38.3(14.1)$ & $45.4(11.8)$ \\
\hline Mental health & 40.0 (11.6) & $43.7(11.0)$ & 47.1 (11.7) & $48.4(11.2)$ \\
\hline
\end{tabular}

were $89.8 \%$ (299 knees) pre-operatively and $83.8 \%$ (279 knees) 12 months after surgery. The proportions of knees with maximum flexion of $135^{\circ}$ were $69.4 \%$ (231 knees) pre-operatively and $38.7 \%$ (129 knees) post-operatively. To ensure that the groups were large enough, we performed a power calculation using the probability of a type 1 error as 0.05 and a power of 0.8 . We found that all the groups had sufficient cases to detect a $10 \%$ difference of maximum points in each parameter, and we assumed this to be clinically significant.

In the comparisons of pre-operative parameters between the subgroups divided by the maximum flexion of $120^{\circ}$, there were no significant differences between the two. In the comparisons of the post-operative parameters, only the social functioning score of SF-36 was significantly higher in the subgroup with maximum flexion of $120^{\circ}$ (41.9 vs 47.3, $\mathrm{p}=0.031)$ and there were no significant differences in the parameters of AKS and WOMAC. Comparisons to find confounders showed that two factors (unilateral or bilateral TKR, and patient height) differed significantly between the groups. The ANCOVA adjustment for height showed no significant effects on the results, and the subgroup analyses for whether unilateral or bilateral TKR was undertaken gave no significant differences.

In the comparisons of pre-operative parameters between the groups divided by the maximum flexion of $135^{\circ}$, the pain and knee scores of AKS had a significant difference (21.4 vs 23.2, $\mathrm{p}=0.032$ and 19.4 s $24.3, \mathrm{p}<0.001$, respectively). Comparisons of the post-operative parameters displayed a significant difference only for the function score of WOMAC (17.5 vs 14.3, p = 0.031). The knee score of AKS showed a difference with statistical tendency (69.9 vs $71.3, p=0.06)$. There were no significant differences in the SF-36 parameters. Comparisons to find confounders revealed that three factors (implant type, weight and BMI) differed significantly between the groups. The ANCOVA adjustment for weight and BMI showed no significant effects on these results. In the subgroup analyses of the three implants (Emotion, Genesis II - CR, Genesis II - PS), all showed similar findings. The difference in function score of WOMAC was greatest in Genesis II CR (21.0 vs 13.6, $\mathrm{p}=0.048)$, followed by Genesis II PS (15.5 vs $12.0, \mathrm{p}=$ 0.207 ) and E-motion (16.5 vs $16.4, \mathrm{p}=0.986)$.

\section{Discussion}

The findings in this study do not support our hypothesis that the post-operative degree of maximum flexion would correlate well with the clinical parameters of established scoring systems for TKR in Korean patients.

We were able to find only two previous studies focusing on the association between maximum flexion and clinical outcome after TKR. ${ }^{12,25}$ A maximum flexion $<70^{\circ}$ is associated with poor walking ability and more than $110^{\circ}$ relates to good walking ability. ${ }^{25}$ In another study, ${ }^{12}$ patients with maximum flexion $<95^{\circ}$ had worse WOMAC function scores than those who had maximum flexion above $95^{\circ}$. However, neither study correlated outcome with highflexion knees $\left(>135^{\circ}\right)$. 
In Western subjects, maximum flexion typically ranges from $100^{\circ}$ to $125^{\circ}$, the proportion of patients with maximum flexion $>120^{\circ}$ is not high ${ }^{6-12}$ and their lifestyles do not require high flexion. ${ }^{3-5}$ Therefore, it may be difficult to perform a study to investigate the correlation of the amount of maximum flexion with clinical outcome in the knees, with maximum flexion in a high flexion range.

In this study, the parameters of three established scoring systems for the knee joint or quality of life were adopted to evaluate the correlation between maximum flexion and clinical outcome. The AKS (physician scoring system) and WOMAC (patient driven) are for joint-specific evaluation and the SF-36 assesses the overall quality of life. Each system therefore has its own characteristics. In the current study, the function score of WOMAC was significantly better for knees with maximum flexion of $135^{\circ}$ ( $\mathrm{p}=0.031$ ). In contrast, the function score of AKS and the corresponding scores of SF-36 (physical function and role physical) did not show any significant correlations or differences in the comparative analyses for either the $120^{\circ}$ or the $135^{\circ}$ subgroups. These findings suggest that maximum flexion beyond a certain range would not make a significant difference to routine daily activities, but would make subtle differences detectable only by the subjective and patient-driven WOMAC system.

Several limitations should be noted in the current study. First, the implants are different from the current highflexion systems. Therefore, it might be inappropriate to extrapolate our findings to include such systems. Although a previous study negated the advantages of high-flexion designs in Korean patients, ${ }^{13}$ our study does not exclude the possibility that our patients might have had different results had high-flexion systems been used. Secondly, we assumed that our subjects would represent the patient population in Asian culture. However, neither evaluation of socioreligious factors nor consideration of individual variations in living style were undertaken and thirdly, the gender of our subjects was not considered. Female dominance is remarkable in Korean patients undergoing $\mathrm{TKR}^{13,29}$ and $94.7 \%$ of our patients were women. This should be considered if our findings are to be extrapolated to a population with a different gender composition. Fourthly, the correlation between post-operative ROM and clinical parameters was analysed 12 months post-operatively, when it is possible that further changes might occur with longer follow-up. However, previous studies report that the ROM and clinical outcomes reach a plateau around one year after surgery, with few clinically significant changes thereafter, ${ }^{11,13,30}$ and our longitudinal analyses confirmed that ROM and the clinical parameters reached a plateau at 12 months postoperatively. Nonetheless, the limitations of the parameters used in the current study should also be recognised. We used passive non-weight bearing ROM and the clinical parameters of AKS, WOMAC and SF-36. Notwithstanding their frequent use, they may not be sensitive enough to detect subtle differences by increased maximum flexion after surgery. Previous studies report the disparity between the active weight-bearing ROM and passive, non-weight bearing ROM. ${ }^{7,10,17}$ The patient's appreciation might be different from that which is evaluated by outcome scores, particularly in the case of Asians, who require frequent high-flexion activities. ${ }^{4,5,31}$ Finally, although we used statistical adjustments to control the effects of many confounding factors, further prospective studies excluding such factors are warranted.

In conclusion, we found that post-operative maximum flexion correlated weakly with a few parameters of clinical outcome, and a few differences were found in the overall quality of life of our patients. These findings may indicate that efforts to increase maximum flexion would not bring enough benefit for Asian patients. Therefore, as high-flexion activities might jeopardise implant survival, we suggest that efforts to increase post-operative maximum flexion should be made with caution until concerns relating to high-flexion activities are sufficiently resolved.

This study was supported by the SNUBH Research Fund (B-0508/009-002). The authors are indebted to Sung Ju Kim (MPH, Department of Infectious Disease Control, Korea Centre for Disease Control and Prevention) for his help with the statistical analyses.

No benefits in any form have been received or will be received from a commercial party related directly or indirectly to the subject of this article.

\section{References}

1. Insall JN, Dorr LD, Scott RD, Scott WN. Rationale of the Knee Society clinical rating system. Clin Orthop 1989;248:13-4.

2. Alicea J. Scoring systems and their validation for the arthritic knee. In: Insall JN, Scott WN, eds. Surgery of the knee. Third ed. Vol. 2. Philadelphia: Churchill Livingstone, 2001:1507-15

3. Laubenthal KN, Smidt GL, Kettelkamp DB. A quantitative analysis of knee motion during activities of daily living. Phys Ther 1972;52:34-43.

4. Noble PC, Gordon MJ, Weiss JM, et al. Does total knee replacement restore normal knee function? Clin Orthop 2005;431:157-65.

5. Weiss JM, Noble PC, Conditt MA, et al. What functional activities are important to patients with knee replacements? Clin Orthop 2002;404:172-88.

6. Schurman DJ, Rojer DE. Total knee arthroplasty: range of motion across five systems. Clin Orthop 2005;430:132-7.

7. Aigner C, Windhager R, Pechmann M, Rehak P, Engeleke K. The influence of an anterior-posterior gliding mobile bearing on range of motion after total knee arthroplasty: a prospective, randomized, double-blinded study. J Bone Joint Surg [Am] 2004;86-A:2257-62.

8. Klein GR, Parvizi J, Rapuri VR, Austin MS, Hozack WJ. The effect of tibial polyethylene insert design on range of motion: evaluation of in vivo knee kinematics by a computerized navigation system during total knee arthroplasty. J Arthroplasty 2004;19:986-91.

9. Argenson JN, Komistek RD, Mahfouz M, et al. A high flexion total knee arthroplasty design replicates healthy knee motion. Clin Orthop 2004;428:174-9.

10. Banks S, Bellemans J, Nozaki $\mathbf{H}$, et al. Knee motions during maximum flexion in fixed and mobile-bearing arthroplasties. Clin Orthop 2003;410:131-8.

11. Ritter MA, Harty LD, Davis KE, Meding JB, Berend ME. Predicting range of motion after total knee arthroplasty: clustering, log-linear regression, and regression tree analysis. J Bone Joint Surg [Am] 2003;85-A:1278-85.

12. Miner AL, Lingard EA, Wright EA, et al. Knee range of motion after total knee arthroplasty: how important is this as an outcome measure? J Arthroplasty 2003;18:286-94.

13. Kim YH, Sohn KS, Kim JS. Range of motion of standard and high-flexion posterior stabilized total knee prostheses: a prospective, randomized study. J Bone Joint Surg [Am] 2005;87-A:1460-5.

14. Huang HT, Su JY, Wang GJ. The early results of high-flex total knee arthroplasty: a minimum of 2 years of follow-up. J Arthroplasty 2005;20:674-9. 
15. Nagura T, Otani T, Sida Y, Matsumoto H, Toyama Y. Is high flexion following total knee arthroplasty safe?: evaluation of knee joint loads in the patients during maximal flexion. J Arthroplasty 2005;20:647-51.

16. Hassaballa MA, Porteous AJ, Newman JH. Observed kneeling ability after total unicompartmental and patellofemoral knee arthroplasty: perception versus reality. Knee Surg Sports Traumatol Arthrosc 2004;12:136-9

17. Dennis DA, Komistek RD, Stiehl JB, Walker SA, Dennis KN. Range of motion after total knee arthroplasty: the effect of implant design and weight-bearing conditions. J Arthroplasty 1998;13:748-52.

18. Kurosaka M, Yoshiya S, Mizuno K, Yamamoto T. Maximizing flexion after total knee arthroplasty: the need and the pitfalls. J Arthroplasty 2002;17(Suppl 1):59-62.

19. Li G, Schule SL, Zayontz SJ, Maloney WJ, Rubash HE. Improving flexion in total knee arthroplasty. In: Callaghan JJ, Rosenberg AG, Rubash HE, Simonian PT, Wickiewicz TL, eds. The adult knee. Vol. 2. Philadelphia: Lippincott Williams and Wilkins, 2003:1233-44.

20. Li G, Most E, Sultan PG, et al. Knee kinematics with a high-flexion posterior stabilized total knee prosthesis: an in vitro robotic experimental investigation. J Bone Join Surg $[A m] 2004 ; 86-A: 1721-9$.

21. Mulholland SJ, Wyss UP. Activities of daily living in non-Western cultures: range of motion requirements for hip and knee joint implants. Int $J$ Rehabil Res 2001;24:191-8.

22. Bellemans J, Robijns F, Duerinckx J, Banks S, Vandenneucker H. The influence of tibial slope on maximal flexion after total knee arthroplasty. Knee Surg Sports Traumatol Arthrosc 2005:13:193-6.
23. Argenson JN, Scuderi GR, Komistek RD, et al. In vivo kinematic evaluation and design considerations related to high flexion in total knee arthroplasty. J Biomech 2005;38:277-84

24. Kanekasu K, Banks SA, Honjo S, Nakata O, Kato H. Fluoroscopic analysis of knee arthroplasty kinematics during deep flexion kneeling. J Arthroplasty 2004:19:9981003

25. Tew M, Forster IW, Wallace WA. Effect of total knee arthroplasty on maximal flexion. Clin Orthop 1989;247:168-74.

26. Bellamy N, Buchanan WW, Goldsmith CH, Campbell J, Stitt LW Validation study of WOMAC: a health status instrument for measuring clinically important patient relevant outcomes to antirheumatic drug therapy in patients with osteoarthritis of the hip or knee. J Rheumatol 1988;15:1833-40.

27. Ware JE Jr, Sherbourne CD. The MOS 36-item short-form health survey (SF-36). I: conceptual framework and item selection. Med Care 1992;30:473-83.

28. Landis JR, Koch GG. The measurement of observer agreement for categorical data. Biometrics 1977;33:159-74.

29. Kim JM, Moon MS. Squatting following total knee arthroplasty. Clin Orthop 1995;313:177-86

30. Aglietti P, Baldini A, Buzzi R, Lup D, Luca L. Comparison of mobile-bearing and fixed-bearing total knee arthroplasty: a prospective randomized study. J Arthroplasty 2005;20:145-53.

31. Kane RL, Saleh KJ, Wilt TJ, Bershadsky B. The functional outcomes of total knee arthroplasty. J Bone Joint Surg [Am]2005;87-A:1719-24. 